\title{
Contemporary hearing aid amplification: issues and outcomes in 2018
}

\begin{abstract}
Vast technological advances and improved clinical outcomes impact contemporary hearing aid fittings. The primary complaint across all hearing aid wearers has traditionally been their ability to understand speech in noise. Previous generations of hearing aids focused on making sounds louder, this made good sense and served as a reasonable starting point. However, simply making sounds louder did not address spatial hearing (i.e., knowing the location from which sounds originate) and did not improve signal to noise ratios. Contemporary commercially available hearing aid fittings successfully address these issues and other aspects of sound reproduction, beyond loudness. In this article, we'll review multiple publications which address benefits and outcomes of contemporary hearing aid amplification and demonstrate clear advantages with regard to satisfaction and improved speech in noise results when fitted properly and professionally delivered.
\end{abstract}

Volume 10 Issue I - 2018

\author{
Douglas L Beck,' Nicholas Le Goff² \\ 'Executive Director of Academic Sciences, Oticon Inc, Somerset, \\ NJ. USA
}

${ }^{2}$ Senior Researcher, Oticon A/S, Smorum, Denmark

Correspondence: Douglas L Beck, Executive Director of Academic Sciences, Oticon Inc, Somerset, NJ. USA, Email Doug.beck@oticon.com

Received: December 12, 2017 | Published: January 29, 2018

Keywords: hearing aid, qol, speech-in-noise, audiogram

Abbreviations: QOL, quality of life; HCPs, hearing care professionals; SIN, speech-in-noise; DNR, digital noise reduction; DI, directivity index; MSAT, multi speaker access technology

\section{Introduction}

Hearing aid fittings, expectations and outcomes have changed dramatically in the last decade. Vast technological advances and improved clinical and diagnostic acumen impact contemporary hearing aid fittings and can improve speech-in-noise ability, ${ }^{1}$ deliver improved sound quality, ${ }^{2}$ maintain spatial cues ${ }^{3}$ and more.

Previous generations of hearing aid fittings focused on hearing thresholds such that hearing thresholds were improved (i.e., making sounds louder) while not exceeding uncomfortable loudness levels. Of course, as hearing loss is often characterized simply by audiograms and by elevated thresholds, and as hearing aids fitting formulas originated in the analog era, improved thresholds made good sense and served as a reasonable starting point.

However, despite sound being "loud enough" the primary complaint of people with hearing loss, and the primary complaint of people with traditional hearing aid amplification, remains their inability to understand speech-in-noise.

Modern commercially available hearing aid fittings successfully address many more aspects of sound reproduction than simple loudness amplification. Indeed, in this article, we'll review studies and questionnaires addressing published benefits and outcomes of contemporary hearing aid amplification. These publications demonstrate clear advantages, satisfaction and improved speech in noise results, when fitted properly and professionally delivered.

\section{Hearing loss and hearing aids: the last 20 years}

The National Council on Aging ${ }^{4}$ assembled data on more than 2300 people with hearing loss and more than 2000 family member reports to measure the effects of untreated hearing loss with regard to quality of life (QOL) and the impact of hearing aid amplification on QOL. The authors compared the perceptions of people with hearing loss to their family members, to identify why people with hearing loss often do not seek treatment. The NCOA study noted older people with hearing loss who do not seek and acquire hearing aid treatment are more likely to report negative effects; sadness, depression, worry, anxiety, paranoia, less social activity, insecurity and emotional turmoil. However, people who seek and acquire hearing aid treatment report better familial relationships, better feelings about themselves, improved mental health, as well as greater independence and security.

Shield ${ }^{5}$ reported international data (primarily on adults) and stated deaf and hard-of hearing $(\mathrm{HOH})$ people are discriminated against at work. She reported evidence (RNID, 2000 and Bradshaw, 2002) that $70 \%$ of deaf and/or $\mathrm{HOH}$ people reported their deafness had prevented them from getting a job, $68 \%$ reported seeking work was a problem, $52 \%$ reported their deafness (or lack of available communication services) prevented them from further training or education, 74\% reported reduced opportunities for promotion due to hearing loss, $60 \%$ noted their colleagues did not understand their hearing issues and $64 \%$ experienced communication barriers at work.

Shield states approximately 1 in 5 people in Europe are hearing impaired and hearing impairment can cause loneliness, depression, and low self-esteem. Shield reports hearing loss impacts family and other close relationships and $\mathrm{HOH}$ people may experience prejudice and abuse, secondary to their disability. She reports $\mathrm{HOH}$ people often deny their problem and delay seeking help. In the UK and Scandinavia (where hearing aids are supported by the government, i.e., free to citizens) fewer than one in three people who might benefit from hearing aids acquires them, and of those who acquire hearing aids, approximately one-third do not use them. Surprisingly, Shield reports these trends and proportions of $\mathrm{HOH}$ people owning and using hearing aids has not changed in 40years despite vast improvements in the appearance, technology and performance of hearing aids. Shield states the perceived benefit provided via amplification is related to the amount of use, rather than the degree of hearing loss. She reports "overwhelming evidence" that hearing aids provide significant improvement in the overall QOL as well as self-confidence, psychological functioning, health, social life and family relationships.

Abrams \& $\mathrm{Kihm}^{6}$ report ten percent of Americans have hearing loss, yet only about $1 / 3$ rd of those with hearing loss acquire hearing 
aids. They report satisfaction has increased to $81 \%$ and of note, for new hearing aids (less than 4 years old) satisfaction is $85 \%$. The rate of non-use (hearing aids owned but not used) has decreased to $3 \%$ and experienced hearing aid wearers report new hearing aids are substantially better than prior models. Further, Abrams and Kihm note satisfaction with professional hearing care providers is about $94 \%$ for those owning hearing aids. They report that for people in the USA with hearing loss, fewer than 1 in 10 have acquired personal sound amplification products (PSAPs, are relatively inexpensive and can be purchased without the benefit or guidance of hearing care professional involvement with regard to testing, diagnosis, fitting, recommendations, guidance and management etc.) Abrams and Kihm report $72 \%$ of hearing aid owners report mild-to-moderate hearing loss.

Valente \& Amlani $^{7}$ report that in the USA, the adoption rate for people with hearing loss who acquire hearing aids is about one-third. They argue cost is not the major barrier. For example, in countries with the highest adoption rates such as Norway (42.5\%), Switzerland $(39 \%)$ and the UK $(41 \%)$, hearing aids are fully or partially subsidized by the government. Yet, almost $60 \%$ of people with hearing loss are unwilling to adopt hearing aids despite no personal monetary cost. Valente and Amlani report that if the USA fully subsidized hearing aids the penetration rate would not be expected to increase by more than $10 \%$. Further, they report a recent study in which more than 600 veterans were screened and determined to have hearing loss. Fewer than one-third complied with a professional recommendation to seek hearing aids and fewer than half of those adopted hearing aids, despite no out-of-pocket expense.

In a recent Cochrane Study, Ferguson et al., ${ }^{8}$ concluded moderate quality (scientific) evidence demonstrates that hearing aids not only improve hearing, but also improves health-related quality of life (QOL). Ferguson and colleagues stated hearing aids are an appropriate intervention and hearing aids are, and should be, the firstline management option for people with hearing loss. Further, when professionals treat hearing loss with hearing aid amplification, the outcomes are beneficial and significantly impact the end-user's QOL.

Ferguson and colleagues reported the "...main clinical intervention for mild to moderate hearing loss is the provision of hearing aids..."and ..."the aim of hearing aid use is to reduce the negative consequences of hearing loss and improve participation in everyday life..." Ferguson and colleagues selected and evaluated five random controlled trials involving 825 participants with mild to moderate hearing loss, participants were assigned to one of three groups; hearing aids, no hearing aids, or placebo hearing aids. Participants were between 69 and 83years of age. The authors report a "large beneficial effect of hearing aids on hearing-specific healthrelated quality of life associated with participation in daily life..."and "a small beneficial effect of hearing aids on general health-related quality of life..." Ferguson and colleagues concluded "hearing aids are effective at improving hearing-specific health-related quality of life, general health-related quality of life and listening ability in adults with mild to moderate hearing loss."

\section{Contemporary amplification -- issues \& outcomes}

As we move through the first half of the 21st century, professional hearing aid fittings are changing and evolving. In brief, hearing can be defined as perceiving sound, and listening can be defined as the ability to comprehend, or makes sense of sound. Tremendous technological advances and sophisticated clinical acumen positively impact contemporary hearing aid fittings with regard to hearing and listening.

Increasingly, hearing care professionals (HCPs) perform Speechin-Noise (SIN) tests to approximate the patient's baseline performance in the most challenging acoustic situation for people with mildmoderate sensorineural hearing loss (i.e., understanding speechin-noise). Some HCPs also employ sophisticated real ear/probemicrophone measures to ascertain the exact acoustic characteristics of sound medial to the tympanic membrane, to assure and maximize audibility and comfort of speech sounds, while not exceeding individual loudness tolerance levels. Contemporary hearing aid fitting factors include the patient's personal sound quality preferences, their age (to approximate neurological processing speed), their pure tone thresholds, their sound field speech-in-noise (SIN) ability (unaided and aided can be evaluated in 5 to 10minutes) and of note, improving the SIN may ultimately be considered a highly pragmatic hearing aid fitting goal, their listening and communication abilites and needs ${ }^{9}$ and may include the results of a cognitive screening for older adults ${ }^{10}$ to better understand and manage the needs of each individual.

Beck and Clark (2009) reported "audition matters more as cognition declines, and cognition matters more as audition declines." They reported patients live in a world where cognition, attention, memory, and hearing interact - and each plays a critical role in listening. They reported when a bottom-up sensory system (i.e, hearing) is compromised, the top-down (i.e., cognitive) system must work harder to make sense of the attenuated or distorted input. Thus, to compensate, the cognitive system "re-allocates resources to increase attention, attends more to context, maximizes short-term memory, and applies knowledge previously acquired. These re-allocations of energy and resources likely slow and reduce processing ability." That is, in the presence of hearing loss, the brain has to work harder to hear, and to listening. Thus, listening effort substantially increases, processing slows, recall is negatively impacted and errors increase.

Beck and Flexer (2011) addressed the differences between hearing and listening. They reported hearing can be measured in the absence of comprehension and it can be measured without volitional attention to sound. However, listening is a learned skill. Listening is the ability to attribute meaning to sound and requires a reasonable signal-tonoise ratio, vocabulary, working memory, the ability to know where to focus one's attention, cognitive ability and more. Beck and Flexer reported listening is a cognitive event and "listening is where hearing meets brain."

Beck \& Le Goff ${ }^{11}$ noted as professionals evolve from traditional hearing-based approaches to focus on listening based-outcomes, professionals facilitate improved outcomes. That is, as HCPs provide enhanced acoustic information, the brain is better able to make sense of sound. BrainHearing ${ }^{\mathrm{TM}}$ is facilitated by helping the brain orient, separate, focus, and recognize sounds - through the use of modern and highly sophisticated hearing aid technologies. Indeed, BrainHearing ${ }^{\mathrm{TM}}$ starts with acknowledging that speech understanding, particularly in challenging listening situations, is a cognitive process.

\section{Contemporary hearing aid fittings}

Beck \& Behrens ${ }^{10}$ reported that although advanced digital noise reduction (DNR) circuits do not improve individual word recognition, they may facilitate cognitive benefits. When using DNR; older children have been shown to learn new words more rapidly, ${ }^{12}$ listening occurs with less effort ${ }^{13}$ and many people recall conversations more accurately with DNR engaged. ${ }^{14}$ Beck \& Behrens, in agreement 
with Dillon, Ching \& Golding..$^{15}$ suggested the "go to" amplification settings for children and adults should include active DNR circuits.

Beck \& Le Goff ${ }^{2}$ noted traditional approaches to noise reduction (DNR and directional microphones) were limited, and the published results were less than the expectations of the wearers. Venema and Beck (2017) reported that although the directivity index (DI) of a particular hearing aid may indicate 4,5 , or $6 \mathrm{~dB}$ directional advantage, it is important to appreciate that the DI is obtained on a "manikin in an anechoic chamber listening to pure-tones in relative silence." They report that in the real world, the actual benefit obtained from directional microphones is often considerably less than the DI.

2017 Beck \& Le Goff ${ }^{2}$ described a new protocol to substantially reduce noise while improving listening ability and maintaining substantial spatial cues. Multi Speaker Access Technology (MSAT) was designed to supplant DNR and directional hearing aid noise reduction systems. MSAT is among the most significant processing advantages incorporated in Oticon $\mathrm{Opn}^{\mathrm{TM}}$.

\section{Contemporary hearing aid fitting outcomes: quantitative outcomes}

Beck \& Porath ${ }^{16}$ report their analysis of 700 hearing aid wearers fitted with MSAT as applied in Oticon $\mathrm{Opn}^{\mathrm{TM}}$. The percentage of wearers who report "satisfied or very satisfied" is $95 \%$ ( $25 \%$ reported satisfied and $70 \%$ reported very satisfied). Satisfaction reports of this magnitude have not been previously reported on such a large sample size. Of note, Abrams \& $\mathrm{Kihn}^{6}$ reported in MarkeTrak 9 (MT9) that overall, $81 \%$ of hearing aid owners were satisfied with their devices and interestingly, for people with hearing aids less than a year old, satisfaction was reported to be $91 \%$.

Chasin ${ }^{2}$ compared ten musicians and ten non-musicians, all of whom were experienced hearing aid wearers. He compared the results of Oticon $\mathrm{Opn}^{\mathrm{TM}}$ to previous technologies and reported the newer technology (Oticon Opn) provides statistically significant improved naturalness and improved clarity (for musicians and non-musicians) while listening to music. Both groups reported speech cues were easier to hear, listening effort was decreased, sounds were more pleasant and the ability to hear speech in quiet, was also improved.

Beck \& Le Goff' ${ }^{1}$ published the results of a sophisticated speechin-noise (SIN) comparison and evaluation procedure. Twenty-five individuals served as listeners and were challenged by three people speaking randomly from multiple locations around the listener in the presence of three noise sources (see illustration one). The protocol was designed to better replicate real-world, challenging listening situations with multiple speakers. Of note, each listener was allowed to turn his/her head as desired to better attend to the person speaking, as they desired.

The authors compared SIN results (and word recognition aka discrimination scores) from three popular technologies; directional, narrow-band directional (i.e., beamforming) and MSAT in a realistic and dynamic, difficult listening scenario (Figure 1).

\section{Performance and results}

While listening to the center talker (talker 2) directly in front of the listener (see Figure 1), the listener's task is relatively easy as the person speaking is located directly in front of the listener, and the listener easily accesses redundant visual and auditory cues. Although the two directional technologies are designed more-or-less for this unique situation (i.e. the listener facing the person talking) it is noteworthy that MSAT performed statistically the same as the best directional technology (see Figure 2) and MSAT outperformed the other directional technology.

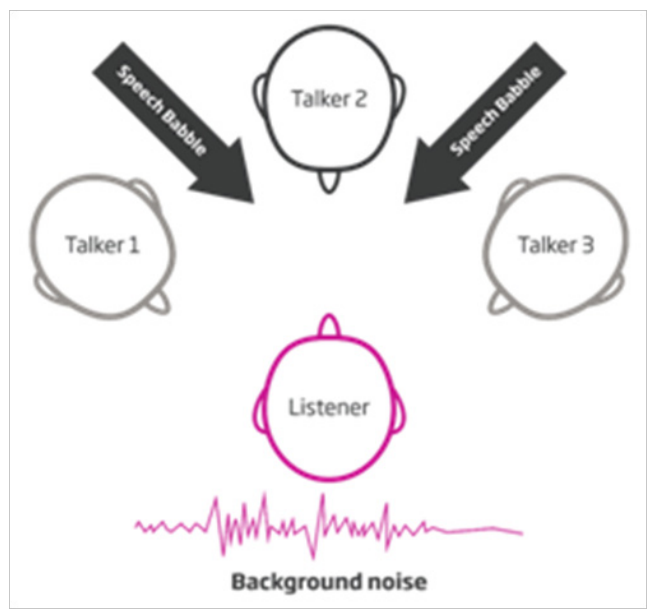

Figure I The Speech In Noise Protocol.

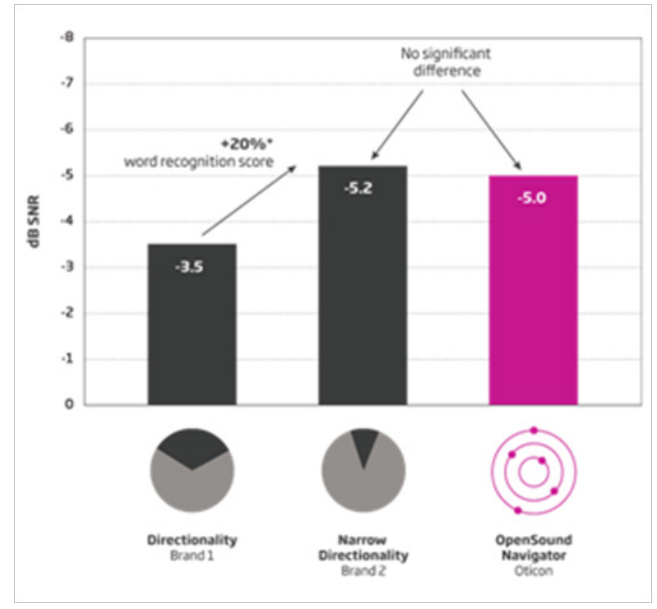

Figure 2 Results obtained when Talker 2 speaks from the center.

SIN results for TALKER TWO/CENTER TALKER (Figure $2 \&$ $3)$.

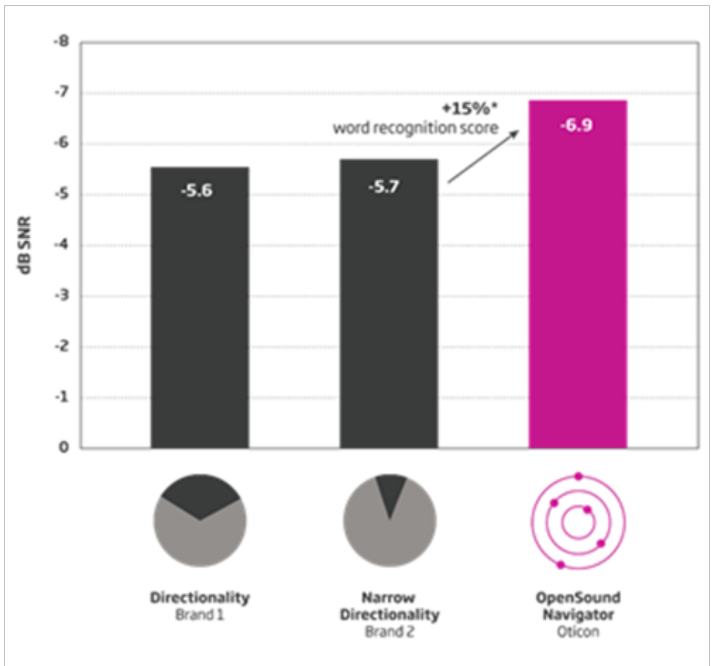

Figure 3 Results obtained when Talkers I and 3 speak from the left and right side. 
As the listener's task becomes more challenging, such as when Talkers 1 and 3 are randomly speaking, the SIN outcomes become more apparent. It can be seen that Directionality and Narrow Directionality lead to an improved SRT by about the same amount (5.65dB, statistically the same). However, the result obtained via MSAT is statistically better than either directional protocol and resulted in an overall performance improvement of $6.9 \mathrm{~dB}$, with regard to the SNR (Figure 4).

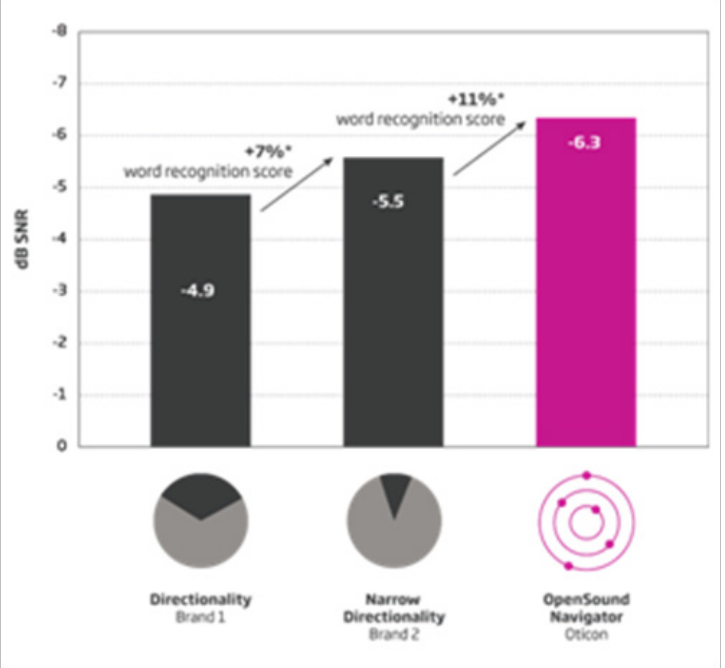

Figure 4 Overall results from Talkers I, 2 and 3 across all three technologies.

In Figure 4, we demonstrate the averages across all listening situations and the functional benefit pattern of the three technologies becomes more apparent. The average SRT (across all listening situations) via Directional is $-4.9 \mathrm{~dB}$. For Narrow Directionality, the average SRT is $-5.5 \mathrm{~dB}$. These two scores $(-4.9$ and -5.5$)$ are statistically different from each other and Narrow Directionality outperforms directional. However, MSAT provides a statistically significant improvement over Narrow Directionality, across all tasks $(-6.3 \mathrm{~dB})$, with concomitant improvement in word recognition scores

\section{Contemporary hearing aid fitting outcomes: qualitative outcomes}

Not only do contemporary hearing aid fittings yield improved consumer satisfaction and improved speech-in-noise results, but these same products score particularly well with regard to surveys of professionals who dispense them.

In a recent (2017) Hearing Tracker/UBS survey of 400 hearing care professionals (http://about-neo.ubs.com/content/research) MSAT technology outperformed all other technologies with specific regard to patient satisfaction, as well as value, sound quality, ease of use and aesthetics.

\section{Summary and discussion}

Contemporary hearing aid fittings outperform traditional technologies with regard to the issues wearers care most about; understanding speech in noise, sound quality, user satisfaction and more.

This article has reviewed previously published results and observations about hearing loss and hearing aids, and has demonstrated the multiple benefits associated with a paradigm shift regarding fitting protocols, advanced technologies and outcomes.
Specifically, although hearing thresholds remain a formidable factor in modern hearing aid fittings, many other factors are now considered and addressed, as what matters most (to wearers and HCPs) are speech in noise results. This article and the publications cited herein demonstrate clear advantages, satisfaction and improved speech in noise results, when contemporary technologies are fitted properly and professionally delivered.

\section{Conflicts of interest statement}

The first author (Beck) declares he is a full-time employee of Oticon Inc., Somerset, NJ, and he receives a salary. The second author (Le Goff) declares he is a full-time employee of Oticon A/S, Smorum, Denmark, and he receives a salary. Neither author reports other financial, non-financial, or other conflicts of interest.

\section{Acknowledgments}

None.

\section{Conflicts of interest}

Author declares there are no conflicts of interest.

\section{Funding}

None.

\section{References}

1. Beck DL, Le Goff N. A Paradigm Shift in Hearing Aid Technology. Hearing Review. 2016.

2. Chasin M. A novel technique to improve amplified sound quality for both music and speech. Hearing Review. 2017;24(8):32-36.

3. Beck DL, Sockalingam R. Facilitating Spatial Hearing Through Advanced Hearing Aid Technology. Hearing Review. 2010.

4. National Council on the Aging. The Consequences of Untreated Hearing Loss in Older Persons. 1999

5. Shield B. Evaluation of the social and economic costs of hearing impairment. A report for Hear-It. 2006.

6. Abrams H, Kihm J. An Introduction to MarkeTrak IX: A New Baseline for the Hearing Aid Market. Hearing Review. 2015

7. Venema T, Beck DL. Interview titled Noise Reduction, Compression for Clinicians, and More. Hearing Review. 2017.

8. Ferguson MA, Kitterick PT, Chong LY, et al. Hearing aids for mild to moderate hearing loss in adults. Cochrane Database of Systematic Reviews. 2017.

9. Beck DL. Best practices in hearing aid dispensing: An interview with Michael Valente, PhD. Hearing Review. 2017;24(12):39-40.

10. Beck DL, Behrens T. The Surprising Success of Digital Noise Reduction. Hearing Review. 2016.

11. Beck DL, LeGoff N. Speech-in-noise test results for Oticon Opn. Hearing Review. 2017;24(9):26-30.

12. Pittman A. Age-related benefits of digital noise reduction for short-term word learning in children with hearing loss. J Speech Lang Hear Res. 2011;54(5):1448-1463.

13. Desjardins JL, Doherty KA. The effect of hearing aid noisereduction on listening effort in hearing impaired adults. Ear Hear. 2014;35(6):600-610.

14. Ng EHN, Rudner M, Lunner T, et al. Effects of noise and working memory capacity on memory processing of speech for hearing aid users. Int J Audiol. 2011;52(7):433-441. 
15. Dillon H, Ching T, Golding M. Heaeing Aids for Infants and Children (Chapter 20). In: Flexer C \& Madell J (Eds.), Pediatric Audiology, Diagnosis, Technology and Management. 2014.
16. Beck DL, Porath M. Consumer Responses to the Oticon Opn ${ }^{\mathrm{TM}}$ Hearing Aid. 2017. 